\title{
Aqueous Humor Biomarkers of Müller Cell Activation in Diabetic Eyes
}

\author{
Stela Vujosevic, ${ }^{1}$ Alessandra Micera,${ }^{2}$ Silvia Bini, ${ }^{1}$ Marianna Berton, ${ }^{1}$ Graziana Esposito, ${ }^{2}$ \\ and Edoardo Midena ${ }^{1,2}$ \\ ${ }^{1}$ Department of Ophthalmology University of Padova, Padova, Italy \\ ${ }^{2}$ Fondazione G. B. Bietti, Istituto di Ricovero e Cura a Carattere Scientifico (IRCCS), Roma, Italy
}

Correspondence: Edoardo Midena, Department of Ophthalmology University of Padova, Via Giustiniani 2 35128 Padova;

edoardo.midena@unipd.it.

Submitted: January 27, 2015

Accepted: April 27, 2015

Citation: Vujosevic S, Micera A, Bini S, Berton M, Esposito G, Midena E. Aqueous humor biomarkers of Müller cell activation in diabetic eyes. Invest Ophthalmol Vis Sci. 2015;56:39133918. DOI:10.1167/iovs.15-16554
Purpose. To identify early biomarkers of retinal Müller cell activation in diabetic eyes with or without clinically detectable signs of diabetic retinopathy (DR).

Methods. This study was a cross-sectional comparative case series. The aqueous humor (AH) of 34 eyes was collected in 12 healthy controls, 11 diabetic patients without DR, and 11 diabetic patients with nonproliferative DR. Full ophthalmic examination and spectral-domain optical coherence tomography were performed in all eyes. Glial fibrillary acidic protein (GFAP), aquaporin 1 (AQP1), and aquaporin 4 (AQP4) were quantified in $\mathrm{AH}$ samples as biomarkers of Müller cell activity by ELISA. Statistical analysis was performed with ANOVA followed by Tukey-Kramer post hoc test.

Results. There was no significant difference in the age among the three groups. Mean concentration of GFAP, AQP1, and AQP4 significantly increased in diabetic eyes versus controls $(P<0.05$, for each comparison). Glial fibrillary acidic protein and AQP1 showed an approximate 2-fold increase, whereas AQP4 showed an approximate 25-fold increase in diabetics with DR versus controls. In diabetics without DR, AQP4 showed an approximate 6fold increase versus controls.

Conclusions. Glial fibrillary acidic protein, AQP1, and AQP4-biomarkers of Müller cell activity-are significantly increased in human eyes with diabetes, confirming that Müller cells are precociously affected by diabetes mellitus.

Keywords: Müller cell, GFAP, aquaporin, diabetes mellitus, diabetic retinopathy
$\mathrm{D}$ iabetic retinopathy (DR) is a frequent complication of diabetes mellitus. ${ }^{1}$ Diabetic retinopathy has a multifactorial pathophysiology as chronic hyperglicemia affects all retinal components causing neuronal cell degeneration, glial cell dysfunction, and microvascular damage. ${ }^{2}$

The main retinal glial cells are Müller cells. Müller cells extend through the entire retina from the photoreceptor inner segments to the internal limiting membrane. Müller cells are neuronal structural support cells and represent connection elements between the neurons and the vascular cells, hence regulating neuronal nutrition, development, and metabolism. Moreover, they contribute to the local inflammatory response. ${ }^{3}$ Müller cells are important in metabolic support and nutrition of neurons; potassium and water homeostasis; protection against oxidative stress; contribution to neuronal signaling; recycling of photopigments; and release of neuroactive and vasoactive substances (increased VEGF/decreased pigment epithelium derived factor, matrix metalloproteinase secretion, and retinal development). ${ }^{4-6}$ Müller cells are particularly susceptible to diabetes-induced damage and are considered as key elements in the onset and the progression of $\mathrm{DR}^{7}$ Müller cells react to hyperglycemic condition by facing a reactive gliosis, a process characterized by three nonspecific responses: hypertrophy, cellular proliferation, and an increase in intermediate filament proteins nestin, vimentin, and GFAP (glial fibrillary acidic protein).
The aquaporins are a family of integral membrane proteins with low molecular weight (approximately $30 \mathrm{kDa} /$ monomer) arranged in tetramers, which mediate the transcellular flow of free water and in some cases of gas and solutes. The aquaporins have a crucial task in the preservation of the ion and osmotic equilibrium inside the central nervous system (CNS), neuroretina included, in response to different osmotic gradients and hydrostatic pressures. $^{8}$ Among the various aquaporins, AQP1 and AQP4 have received the greatest attention, because it has been demonstrated that in the retina, they facilitate the bidirectional flow of free water and also modulate the neuronal excitability. ${ }^{8}$

The purpose of this study was to quantify GFAP, AQP1, and AQP4 in the aqueous humour $(\mathrm{AH})$ of diabetic patients without DR and with nonproliferative DR (NPDR), as biomarkers of early Müller cell activation.

\section{Materials ANd Methods}

\section{Study Population}

Thirty-four eyes of 34 patients appointed for cataract surgery were evaluated at the Department of Ophthalmology, University of Padova. The study population consisted of a control group (12 healthy subjects); 11 diabetic patients without diabetic retinopathy (no DR); and 11 diabetic patients with nonproliferative DR. 
Inclusion criteria were indication for cataract surgery and presence of diabetes mellitus type 2 in diabetic patients (according to the World Health Organization criteria). ${ }^{9}$ The major exclusion criteria in all groups were presence of any neurodegenerative disease (e.g., Alzheimer, Parkinson, etc.); systemic or ocular therapy with steroids; anti-VEGF drugs in the 3 months preceding the study; intraocular pressure $\geq 22$ $\mathrm{mm} \mathrm{Hg}$ or a history of glaucoma; other ocular disease of vascular, degenerative, or inflammatory nature not related to DM; a history of eye surgery in the 3 months before sampling; and presence of macular edema.

Informed consent was obtained from each patient and the research was carried out in accordance with the tenets of the Declaration of Helsinki regarding experimentation involving human tissue. Local ethics committee approval for the study was obtained.

\section{Study Design}

This study was a cross-sectional, case-control series evaluation. Each subject underwent a complete ophthalmologic examination with color fundus photos and spectral-domain optical coherence tomography (OCT) exam of the studied eye in order to exclude the presence of macular edema. The presence of DR was graded according to internationally established criteria on color fundus photos. ${ }^{10}$

\section{Sampling, Storage, and Total Protein Analysis of Aqueous Humour}

All patients underwent standard preoperative disinfection and preparation for cataract surgery. We aspirated AH (150-200 $\mu \mathrm{L})$ from the anterior chamber of the eye, via parecentesis under microscope, using a 30-gauge needle and an insulin syringe (1 $\mathrm{mL}$ ), avoiding any contact with the intraocular tissues. Three 1.7-mL microcentrifuge tubes (Starlab Intl. GmbH, Hamburg, Germany) precoded with the patient identification code of the study subject ("PT: ID code") were prepared for each patient/ sampling. The first contained $50 \mu \mathrm{L}$ modified RIPA buffer ( 25 $\mathrm{mM}$ Tris-buffer, $150 \mathrm{mM} \mathrm{NaCl}, 0.1 \%$ Tween20, $1 \mathrm{mM}$ EDTA, $0.1 \%$ SDS, $10 \mathrm{mM} \mathrm{NaF}, 1 \mathrm{mM}$ PMSF, $\mathrm{pH} 7.5$ ); the second contained $50 \mu \mathrm{L}$ extraction reagent (T-PER; Pierce Biotechnology, Rockford, IL, USA), both supplemented with a cocktail of protease inhibitors (\#0078510; Pierce Biotechnology); and the third contained $3 \mu \mathrm{L}$ protease inhibitor. The collected $\mathrm{AH}$ sample from each patient was divided into two $50-\mu \mathrm{L}$ aliquots that were placed into the first and second microcentrifuges. The remaining $\mathrm{AH}$ was placed into the third microcentrifuge. The samples were first stored at $-70^{\circ} \mathrm{C}$ and thereafter shipped to the G.B. Bietti Foundation Istituto di Ricerca e Cura a Carattere Scientifico (IRCCS) for analysis, in accordance with the international regulations regarding the transport of biological samples. At the laboratory unit, $\mathrm{AH}$ samples were sonicated (VibraCell; Sonics, Newton, CT, USA) and debris were removed by centrifugation $(16,162 \mathrm{~g} / 7 \mathrm{~min})$. The total protein content was evaluated using the Bradford method (RIPA) or A280 NanoDrop (TPER) analyses. The protein concentration was calculated based on the linearized standard curve provided by the standard (BSA) or the software (A280). Total protein data was used to detect variations in total protein contents among subgroups and for proper normalization.

\section{ELISA for GFAP, AQP1, and AQP4}

Glial fibrillary acidic protein (NS830; Millipore, Merck KGaA, Darmstadt, Germany); AQP1 (MBS700396; MyBiosource, San Diego, CA, USA); and AQP4 (MBS705290; MyBiosource) were quantified by using commercial high-sensitive ELISA assays, according to manufacturer's suggestions with minor modifications. Briefly, both prediluted samples and standard curve, prepared in TBS buffer (20 mM Tris-Cl and $150 \mathrm{mM} \mathrm{NaCl}$ ) containing $3 \%$ of BSA, $5 \mathrm{mM}$ EDTA, and $1 \mathrm{X}$ protease inhibitor cocktail, were applied to 96-well precoated plates. Standards and detection limits were as follows: 1.5 to $100 \mathrm{ng} / \mathrm{mL}$ and 1.5 ng/mL for GFAP; 15.6 to $1000 \mathrm{pg} / \mathrm{mL}$ and less than $6.6 \mathrm{pg} / \mathrm{mL}$ for AQP4; and 15.6 to $1000 \mathrm{pg} / \mathrm{mL}$ and less than $3.9 \mathrm{pg} / \mathrm{mL}$ for AQP1. Biotin-coupled antibodies and horseradish peroxidasestreptavidin specific bindings were developed with a ready-toused tetramethylbenzidine substrate (eBioscience, San Diego, CA, USA). The colorimetric signal was quantified using an ELISA reader (Sunrise; Tecan Group Ltd., Männedorf, Switzerland). The data $\left(\mathrm{OD}_{\lambda 490-560 \mathrm{~nm}}\right)$ were normalized to total protein content as assessed at the beginning (NanoDrop; Thermo Fisher Scientific, Inc., Waltham, MA, USA). The specific protein concentrations were calculated on the linearized standard curves provided at the end of ELISA.

\section{Statistical Analysis}

Total protein, GFAP, AQP1, and AQP4 concentrations were expressed as mean \pm standard deviation $(\mathrm{mg} / \mathrm{mL}$ or $\mathrm{pg} / \mu \mathrm{g})$. The difference in mean protein concentration, GFAP, AQP1, and $\mathrm{AQP} 4$ concentration among the three groups of patients was compared using the ANOVA test followed by the TukeyKramer post hoc analysis. First, all diabetics were compared with controls. Second, diabetics were subdivided in those without DR and with DR, and separately compared with controls. Kendall Rank coefficient was used for evaluation of AQP4/AQP1 correlation. A value of $P \leq 0.05$ was considered statistically significant. All the analyses were performed using statistical software (SAS 9.3; SAS Institute, Cary, NC, USA) on personal computer.

\section{Results}

\section{Population}

Twelve healthy subjects (controls) consisting of five males and seven females, mean age $75.4 \pm 6.4$ years, (range, $66-87$ years) and 22 diabetics: 11 without DR ( 3 males and 8 females; mean age $73.9 \pm 9.0$ years; range, 56-86 years) and 11 diabetics with DR (6 males and 5 females, (mean age: $69.9 \pm 11.4$ years; range, 49-83 years) were analyzed. There was no significant difference in mean age among the three groups. Mean duration of diabetes mellitus was significantly different between diabetics without DR $7.7 \pm 5.1$ years, (range, 2-15 years), and diabetics with DR: $20.1 \pm 9.8$ years, (range, 10 - 40 years; $P$ $<0.05$ ).

In the control group, mean intraocular pressure (IOP) was $16.3 \pm 2.3 \mathrm{~mm} \mathrm{Hg}$ (range, $12-20 \mathrm{~mm} \mathrm{Hg}$ ) and mean BCVA was $0.41 \pm 0.22 \log$ MAR (range, +1.0 and $+0.15 \log$ MAR). In diabetics without DR, mean IOP was $17.5 \pm 2.4 \mathrm{~mm} \mathrm{Hg}$ (range, 14-22 mm Hg) and mean BCVA was $0.58 \pm 0.52$ logMAR (range, +1.0 and $+0.1 \log$ MAR). In diabetics with DR, mean IOP was $15.7 \pm 2.6 \mathrm{~mm} \mathrm{Hg}$, (range, 11-18 $\mathrm{mm} \mathrm{Hg}$ ) and mean BCVA was $0.63 \pm 0.51 \log$ MAR, (range, +1.52 to +0.15 $\operatorname{logMAR}$ ). There was no significant difference in mean BCVA and IOP among the three groups. In the diabetic retinopathy group, three patients had mild NPDR and eight moderate NPDR.

In all 34 cases, collection of the AH was successful, without contamination by blood or by tissues from the anterior chamber of the eye. The quantity of the AH collected was superior to $150 \mu \mathrm{L}(150-200 \mu \mathrm{L})$. No intraoperative complica- 
TABLE. Retinal Glial Proteins in the Aqueous Humor

\begin{tabular}{|c|c|c|c|c|c|}
\hline \multirow[b]{2}{*}{ Protein } & \multicolumn{5}{|c|}{ Mean Concentration $\pm \mathrm{SD}, \mathrm{pg} / \mu \mathrm{g}$} \\
\hline & Controls, $n=12$ & All Diabetics, $n=22$ & Diabetics w/o DR, $n=11$ & Diabetics With DR, $n=11$ & ANOVA $P$ Value* \\
\hline GFAP & $149.86 \pm 40.73$ & $225.17 \pm 174.99$ & $165.61 \pm 47.19$ & $324.44 \pm 262.55 \dagger \neq$ & 0.02 \\
\hline AQP1 & $50.92 \pm 36.52$ & $50.81 \pm 40.30$ & $28.84 \pm 11.88$ & $105.72 \pm 27.15+\delta$ & $<0.01$ \\
\hline AQP4 & $33.58 \pm 15.75$ & $391.20 \pm 360.68 \|$ & $206.87 \pm 193.799$ & $852.03 \pm 188.94+\S$ & $<0.01$ \\
\hline
\end{tabular}

Comparison of mean concentrations and their standard deviations (in $\mathrm{pg} / \mu \mathrm{g}$ ) of retinal glial proteins: GFAP, AQP1, and AQP4 present in aqueous humor of healthy controls, all diabetics (with and without diabetic retinopathy); diabetics with DR; and diabetics w/o DR.

* One-way ANOVA: comparison among controls, diabetics without DR, and diabetics with DR comparison versus controls

† Tukey-Kramer post hoc test, $P<0.01$.

‡ Comparison versus diabetics without DR, Tukey-Kramer post hoc test, $P<0.05$.

$\S$ Comparison versus diabetics without DR, Tukey-Kramer post hoc test, $P<0.01$.

$\|$ ANOVA, $P<0.01$.

II Tukey-Kramer post hoc test, $P<0.05$.

tion was registered, and the postoperative management was regular in all cases.

\section{Total Protein, GFAP, AQP1, and AQP4 Analyses}

There was no significant difference in mean protein concentration in the $\mathrm{AH}$ among controls, diabetics without $\mathrm{DR}$, and diabetics with DR $(0.44 \pm 0.07 \mathrm{mg} / \mathrm{mL}$ versus $0.45 \pm 0.10 \mathrm{mg}$ / $\mathrm{mL}$ versus $0.46 \pm 0.08 \mathrm{mg} / \mathrm{mL}, P=0.21$; ANOVA test).

There was no significant difference in mean GFAP concentration in the AH between diabetics (with and without DR) and controls $(225.17 \pm 174.99 \mathrm{pg} / \mu \mathrm{g}$ versus $149.86 \pm$ $40.73 \mathrm{pg} / \mu \mathrm{g} ; P=0.15$; ANOVA test).

There was significant difference in mean GFAP concentration in the AH among diabetics with DR, diabetics without DR, and controls (ANOVA, $P=0.02$ ). Mean GFAP concentration significantly increased in diabetics with DR compared both with controls $(324.44 \pm 262.55 \mathrm{pg} / \mu \mathrm{g}$ versus $149.86 \pm 40.73$ $\mathrm{pg} / \mu \mathrm{g}$; Tukey-Kramer post hoc test $P=0.03$ ) and diabetics without DR $(324.44 \pm 262.55 \mathrm{pg} / \mu \mathrm{g}$ versus $165.61 \pm 47.19$ $\mathrm{pg} / \mu \mathrm{g}$; Tukey-Kramer post hoc test $P=0.04$; Table, Fig. 1 fold changes).

There was no significant difference in mean AQP1 concentration in the $\mathrm{AH}$ between diabetics and controls $(50.81 \pm 40.30 \mathrm{pg} / \mu \mathrm{g}$ versus $50.92 \pm 36.52 \mathrm{pg} / \mu \mathrm{g} ; P=0.99$; ANOVA test).

There was significant difference in mean AQP1 concentration in the AH among diabetics with DR, diabetics without DR, and controls (ANOVA, $P<0.01$ ). Mean AQP1 concentration significantly increased in diabetics with DR compared with

GFAP

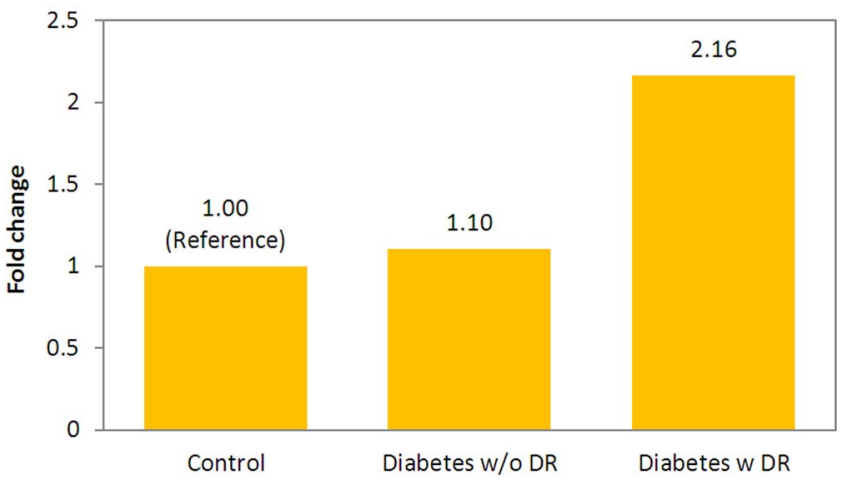

Figure 1. Fold changes of GFAP expression in all groups compared with controls. Positive fold changes indicate an increase in concentration. w/o DR, diabetics without DR; w DR, diabetics with DR. both controls $(105.72 \pm 27.15 \mathrm{pg} / \mu \mathrm{g}$ versus $50.92 \pm 36.52$ $\mathrm{pg} / \mu \mathrm{g}$; Tukey-Kramer post hoc test $P<0.01$ ) and diabetics without DR $(105.72 \pm 27.15 \mathrm{pg} / \mu \mathrm{g}$ versus $28.84 \pm 11.88 \mathrm{pg} /$ $\mu \mathrm{g}$; Tukey-Kramer post hoc test, $P<0.01$; Table, Fig. 2 fold changes).

Concentration of AQP4 in the AH was significantly increased in diabetics versus controls $(391.20 \pm 360.68 \mathrm{pg} /$ $\mu \mathrm{g}$ versus $33.58 \pm 15.75 \mathrm{pg} / \mu \mathrm{g} ; P<0.01$; ANOVA test).

There was significant difference in mean AQP4 concentration in the $\mathrm{AH}$ among diabetics with $\mathrm{DR}$, diabetics without $\mathrm{DR}$, and controls (ANOVA, $P<0.01$ ). Mean AQP4 concentration significantly increased in diabetics without DR compared with controls $(206.87 \pm 193.79 \mathrm{pg} / \mu \mathrm{g}$ versus $33.58 \pm 15.75 \mathrm{pg} / \mu \mathrm{g}$; Tukey-Kramer post hoc test, $P<0.05)$. A significant increase was found in diabetics with DR compared with both controls $(852.03 \pm 188.94 \mathrm{pg} / \mu \mathrm{g}$ versus $33.58 \pm 15.75 \mathrm{pg} / \mu \mathrm{g}$; TukeyKramer post hoc test, $P<0.01)$ and to diabetics without DR $(852.03 \pm 188.94 \mathrm{pg} / \mu \mathrm{g}$ versus $206.87 \pm 193.79 \mathrm{pg} / \mu \mathrm{g}$; Tukey-Kramer post hoc test, $P<0.01$; Table, Fig. 3 fold changes).

The ratio of AQP4 over AQP1 was significantly higher in diabetics compared with controls $(9.77 \pm 9.28$ vs. $0.75 \pm$ $0.25, P<0.01 ;$ ANOVA test). The ratio was significantly different between controls and diabetics without DR $(0.75 \pm$ 0.25 vs. $10.44 \pm 11.28$, Tukey-Kramer post hoc test, $P<0.01$ ) and controls and diabetics with DR $(0.75 \pm 0.25$ vs. $8.10 \pm$ 0.29 , Tukey-Kramer post hoc test, $P<0.05$; Fig. 4).

The Kendall rank analysis showed weak and not significant correlation ( $\tau=0.33, P=0.30)$ between AQP4 and AQP1 in diabetics.

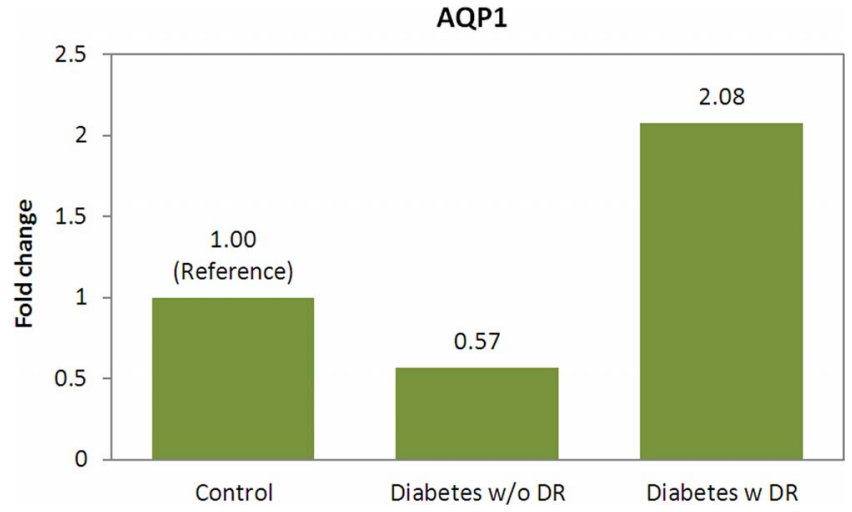

FIGURE 2. Fold changes of AQP1 expression in all groups compared with controls. Positive fold changes indicate an increase in concentration. 


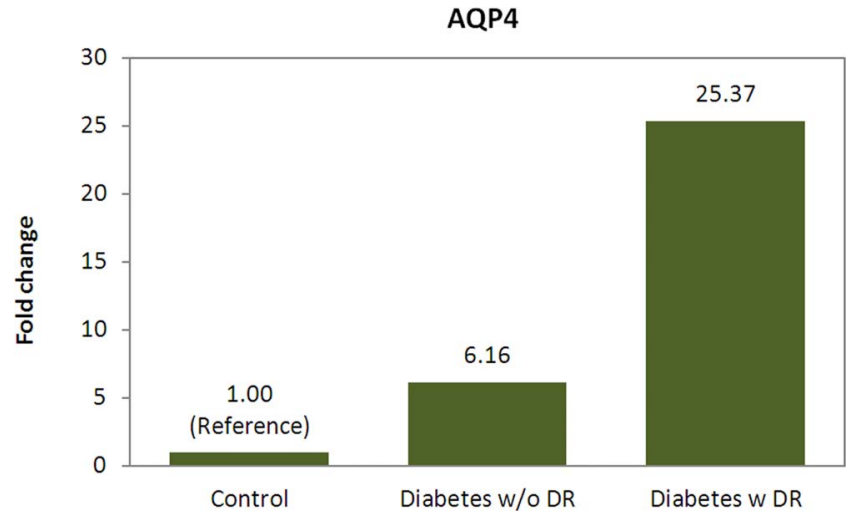

FIgURE 3. Fold changes of AQP4 expression in all groups compared with controls. Positive fold changes indicate an increase in concentration.

\section{Discussion}

To the best of our knowledge, this is the first study to report the aqueous humour concentration of retinal glial proteins GFAP, AQP1, and AQP4 in diabetic eyes with and without nonproliferative retinopathy. An increase in the AQP4, AQP1, and GFAP was documented in diabetic patients.

Mean concentration of GFAP is significantly increased in diabetic patients with DR compared with controls and diabetics without DR, with a mean change of 1.1- to 2.2-fold increase. In normals, GFAP is prevalently expressed by retinal astrocytes and it is therefore detectable only in the retinal nerve fiber layer (RNFL) and ganglion cell layer (GCL), while it is absent in Müller cells. ${ }^{11}$ In animal and human models of DM, GFAP is hyperexpressed by Müller cells, ${ }^{11,12}$ whereas its expression decreases to null in astrocytes. ${ }^{11}$ Barber et al. ${ }^{11}$ demonstrated the increase in GFAP production only in Müller cells, and its reduced expression by astrocytes (preceding the before mentioned increase in Müller cells) in diabetic mice. The same authors detected that a remarkable reduction of GFAP in astrocytes disappears after the administration of insulin. ${ }^{11}$ The increase in GFAP concentration is considered a common marker for reactive gliosis and an early sensor for retinal stress. ${ }^{6,13}$ We have evaluated the concentration of GFAP, in vivo, in $\mathrm{AH}$ both in normal conditions and in the course of DM. At early stages, in diabetics without DR there was no significant change in GFAP versus controls, whereas in diabetics with DR, there is a significant increase in GFAP. These data confirm that GFAP increase is secondary to a significant activation of Müller cells, and therefore may be a later biomarker of Müller cell activation. Different experimental studies have confirmed such hypothesis, suggesting that the glial activation may be responsible for the establishment of functional and metabolic imbalances of the retina causing both neuronal and microvascular damage ${ }^{14-17}$ The increase of GFAP in $\mathrm{AH}$ may be the consequence of macroglial cell activation, namely Müller cell activation. ${ }^{12,16-19}$

There are very limited data in the literature about in vivo evaluation of aquaporins in ocular fluids. In normal conditions, fluids that accumulate inside the neuroretina and in subretinal space are spilled out into the blood via transcellular transport, osmotically coupled with the flow of various molecules, especially ions, at the level of the RPE and Müller cells. Aquaporins facilitate the transcellular water flow. Müller cells regulate the flow of potassium released by active neurons, which if present in excess on the extracellular level may induce neuronal excitability. In this study, AQP4 resulted significantly increased in diabetic patients, even in those

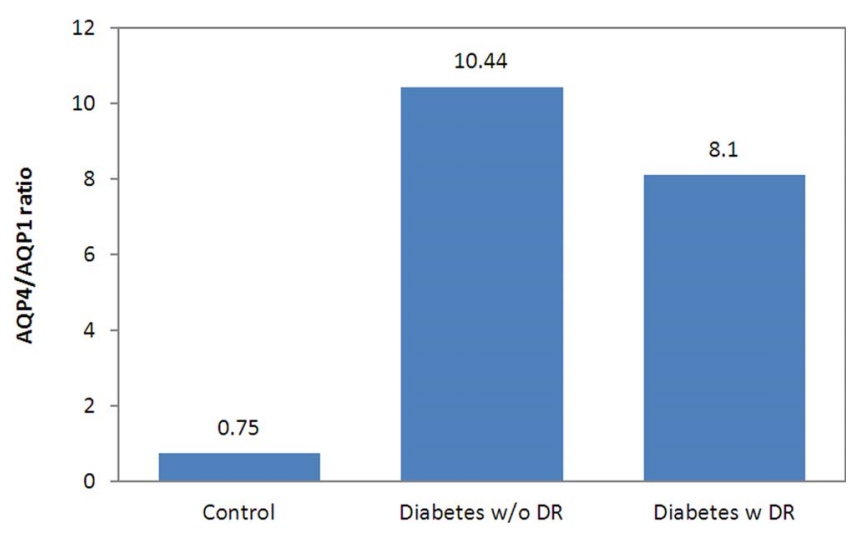

FIgURE 4. Aquaporin 4 (AQP4) and AQP1 ratios in all groups.

without clinical signs of retinopathy (6-fold increase), versus healthy subjects. In diabetic patients with retinopathy, AQP4 was increased by approximately 25 -fold versus healthy subjects. When diabetic patients with and without DR were compared, there was a 4-fold increase in those with DR.

Aquaporin 4 is a channel protein that allows the flow of free water through the cell membrane. ${ }^{20}$ In the retina, AQP4 is normally expressed by the Müller cells and astrocytes. ${ }^{21,22}$ Due to its ability to transport free water and its functional pairing with $\mathrm{K}+$ channels, AQP4 maintains the osmotic balance between the intra- and extracellular matrixes, thus guaranteeing optimal cell functioning. ${ }^{23}$ Zhang et al., ${ }^{24}$ and Cui et al. ${ }^{25}$ have recently provided evidence, in the rat retina, that there is an increase in AQP4 during DM, specifically in Müller cells and astrocytes. While different histological studies have been done regarding the variation in $\mathrm{AQP} 4$ expression in the retina during $\mathrm{DM}$, no data on $\mathrm{AQP} 4$ concentration in human ocular fluids is available so far. ${ }^{7,24-27}$ In the present study, AQP4 concentration was significantly increased not only in the presence of clinical microvascular alterations typical of DR, but also in its preclinical stage. Since AQP4 is expressed in the retina, selectively in the retinal glial cells, it is possible to consider this molecule as an early and effective marker of retinal Müller cell alteration during the course of DM, even without clinical signs of DR, as astrocyte loss had already occurred (experimental studies in diabetic rat retina show a significant loss of astrocytes as early as 4 weeks after the onset of streptozotocininduced diabetes). ${ }^{28}$

Moreover, we have also detected a significant increase in AQP1 in diabetics with retinopathy compared to diabetics without DR ( $\sim 1.4$-fold increase) and healthy subjects ( $\sim 2$-fold increase). As aquaporin 4, the function of AQP1 is also involved in the regulation of the transcellular flow of free water. ${ }^{23}$ Several histological studies analyzed AQP1 expression changes, both in animal and human tissue, during the course of DM. ${ }^{8,27,29,30}$ Gene expression of AQP1 was found upregulated in the retinas of diabetic rats. ${ }^{31}$ Additionally, AQP1 was present in the inner retinal layers (whereas in control tissues, it is normally expressed in the outer retina by photoreceptors and amacrine cells), in particular in Müller cells; and the substitution of AQP4 with AQP1 in retinal surface vessels was found, entailing an increase in its production in streptozotocin-induced diabetic rats. ${ }^{21}$ Perivascular AQP4 expression was reported to be reduced in the superficial vessel plexus, and not altered around the deep vessels in the inner nuclear layer. ${ }^{21}$ Such switch in localization of AQP1 and AQP4 would suggest that the glial cell-mediated water transport in the retina is altered especially at the superficial layer plexus. ${ }^{8,21}$ Even though many studies evaluated different expressions of AQP1 during DM on histological specimens, 
there are no data on concentrations of this protein in ocular fluids. We have examined AQP1 concentrations in the AH of both healthy subjects and diabetics, evidencing the increase of this protein in diabetics with DR. Since we have not found an increase in AQP1 in diabetic patients without DR compared with controls, it is possible to consider that such increase can be detected only when the retinal damage is clinically evident and may be the sign of glial cell modification subsequent to those previously mentioned. Therefore, AQP1 could become a useful marker of glial activation, capable of indicating the progression of retinal damage during the course of DM.

Müller cells become particularly susceptible to diabetesinduced damage and are recognized today as key elements in the onset and the progression of diabetic retinopathy. Müller cells and astrocytes, besides supporting the synaptic activity, maintain the integrity of the blood-retinal barrier and regulate the vasoconstriction related to neuronal activity. However, they react in different ways with regard to hyperglycemic damage: while Müller cells face gliosis, functional activation, and cell proliferation (with consequent relative increase of their number), few studies have analyzed the alterations of the astrocytes, though it seems that there is a significant loss and a functional alteration of these cells, previous to the events relative to Müller cells. ${ }^{28}$ Reactive gliosis of Müller cells possesses both cytoprotective and cytotoxic effects with respect to retinal neurons: mostly in the initial stages of DM, it is neuroprotective and may be interpreted as an effort to limit the extension of tissue damage. Later, in the course of $\mathrm{DM}$, it contributes to neurodegeneration and prevents tissue restoration and normal neuroregeneration. ${ }^{5}$

Glial activation detected during the course of DM is also confirmed by clinical studies with OCT analysis of retinal layer thickness. In a recent study, a significant increase in the thickness of the inner nuclear layer (mostly composed by the nuclei of bipolar and Müller cells) was described in patients with non-NPDR. ${ }^{32}$ The increase in thickness in inner nuclear layer may represent a clinical sign of Müller cell activation due to hypertrophy of these cells. ${ }^{32}$ Therefore, data from the present study may confirm early activation and dysfunction of glial cells in diabetics, especially Müller cells, known as "the communicator" cell between vessels and neurons and responsible for the upregulation of GFAP and AQP4. ${ }^{6,11,12}$

The major limit of this study is the limited number of examined eyes, and the lack of direct correlation with histopathologic results. Therefore, a larger population study would be suggested to increase robustness of this data. Specific retinal histopathologic data in human DM, in earlier stages of $\mathrm{DR}$, is probably impossible to be obtained, mainly for ethical reasons. Notwithstanding, this is the first study that determined in vivo changes in AQP1, AQP4, and GFAP concentrations in $\mathrm{AH}$ in diabetic patients.

In conclusion, during the course of diabetes mellitus, there is a significant increase in the $\mathrm{AH}$ of the protein biomarkers of retinal (macro)glial cell activation, not only when diabetic retinopathy is clinically detectable, but also in its subclinical and early clinical stages. These molecules, easily detected in AH samples, may represent new biomarkers of retinal changes in diabetic patients.

\section{Acknowledgments}

The authors thank Fabiano Cavarzeran, ScD (Department of Ophthalmology University of Padova), for help with statistical analyses and interpretation of data during the revision of the manuscript.

Supported by the Ministry of Health and Fondazione Roma (GB Bietti Foundation, IRCCS).
Disclosure: S. Vujosevic, None; A. Micera, None; S. Bini, None; M. Berton, None; G. Esposito, None; E. Midena, None

\section{References}

1. King H, Aubert RE, Hermann WH. Global burden of diabetes, 1995-2025: prevalence, numerical estimates and projections. Diabetes Care. 1998;21:1414-1431.

2. Antonetti DA, Klein R, Gardner TW. Diabetic retinopathy. $N$ Engl J Med. 2012;366:1227-1239.

3. Newman EA. Müller cells and the retinal pigment epithelium. In: Albert DM, Jakobiec FA, eds. Principles and Practice of Ophthalmology. Philadelphia: W.B. Saunders Company; 1994: 398-419.

4. Bringmann A, Pannicke T, Grosche J, et al. Müller cells in the healthy and diseased retina. Prog Retin Eye Res. 2006;25:397424.

5. Bringmann A, Iandiev I, Pannicke T, et al. Cellular signaling and factors involved in Müller cell gliosis: neuroprotective and detrimental effects. Prog Retin Eye Res. 2009;28:423-451.

6. Bringmann A, Wiedemann P. Müller glial cells in retinal disease. Ophthalmologica. 2012;227:1-19.

7. Curtis T, Hamilton R, Yong PH, et al. Müller glial dysfunction during diabetic retinopathy in rats is linked to accumulation of advanced glycation end-products and advanced lipoxidation end-products. Diabetologia. 2011;54:690-698.

8. Fukuda M, Nakanishi Y, Fuse M, et al. Altered expression of aquaporins 1 and 4 coincides with neurodegenerative events in retinas of spontaneously diabetic Torii rats. Exp Eye Res. 2010;90:17-25.

9. American Diabetes Association. Diagnosis and classification of diabetes mellitus. Diabetes Care. 2012;35:S64-S71.

10. Wilkinson CP, Ferris FL III, Klein RE, et al. Proposed international clinical diabetic retinopathy and diabetic macular edema disease severity scales. Ophthalmology. 2003;110: 1677-1682.

11. Barber AJ, Antonetti DA, Gardner TW. Altered expression of retinal occludin and glial fibrillary acidic protein in experimental diabetes. Invest Ophthalmol Vis Sci. 2000;41:35613568.

12. Mizutani M, Gerhardinger C, Lorenzi M. Müller cell changes in human diabetic retinopathy. Diabetes. 1998;47:445-449.

13. Sarthy V. Focus on molecules: glial fibrillary acidic protein (GFAP). Exp Eye Res. 2007;84:381-382.

14. Van Dijk HW, Verbraak FD, Kok PHB, et al. Early neurodegeneration in the retina of type 2 diabetic patients. Invest Ophthalmol Vis Sci. 2012;53:2715-2719.

15. Gardner T, Antonetti D, Barber A, LaNoue K, Levison S. Diabetic retinopathy-more than meets the eye. Surv Ophthalmol. 2002;47:253-262.

16. Carrasco E, Hernández C, Miralles A, Huguet P, Farrés J, Simó R. Lower somatostatin expression is an early event in diabetic retinopathy and is associated with retinal neurodegeneration. Diabetes Care. 2007;30:2902-2908.

17. Carrasco E, Hernández C, De Torres I, Farrés J, Simó R. Lowered cortistatin expression is an early event in the human diabetic retina and is associated with apoptosis and glial activation. Mol Vis. 2008;14:1496.

18. Lieth $\mathrm{E}$, Barber $\mathrm{AJ}, \mathrm{Xu} \mathrm{B}$, et al. Glial reactivity and impaired glutamate metabolism in short-term experimental diabetic retinopathy. Penn State Retina Research Group. Diabetes. 1998; 47:815-820.

19. Abu-El-Asrar AM, Dralands L, Missotten L, Al-Jadaan IA, Geboes $\mathrm{K}$. Expression of apoptosis markers in the retinas of human subjects with diabetes. Invest Ophthalmol Vis Sci. 2004;45: 2760-2766. 
20. Huber VJ, Tsujita M, Nakada T. Aquaporins in drug discovery and pharmacotherapy. Mol Aspects Med. 2012;33:691-703.

21. Iandiev I, Pannicke T, Reichenbach A, Wiedemann P, Bringmann A. Diabetes alters the localization of glial aquaporins in rat retina. Neurosci Lett. 2007;421:132-136.

22. Iandiev I, Pannicke T, Hollborn M, et al. Localization of glial aquaporin- 4 and Kir 4.1 in the light-injured murine retina. Neurosci Lett. 2008;434:317-321.

23. Castle NA. Aquaporins as targets for drug discovery. Drug Discov Today. 2005;10:485-493.

24. Zhang Y, Xu G, Ling Q, Da C. Expression of aquaporin 4 and Kir4.1 in diabetic rat retina: treatment with minocycline. $J$ Int Med Res. 2011;39:464-479.

25. Cui B, Sun J, Xiang F, Liu L, Li W. Aquaporin 4 knockdown exacerbates streptozotocin-induced diabetic retinopathy through aggravating inflammatory response. Exp Eye Res. 2012;98:37-43.

26. Gerhardinger C, Costa MB, Coulombe MC, Toth I, Hoehn T, Grosu P. Expression of acute-phase response proteins in retinal Muller cells in diabetes. Invest Ophthalmol Vis Sci. 2005;46: 349-357.
27. Fukuda M, Naka M, Mizokami J, Negi A, Nakamura M. Diabetes induces expression of aquaporin-0 in the retinal nerve fibers of spontaneously diabetic Torii rats. Exp Eye Res. 2011;92:195201.

28. Ly A, Yee P, Vessey KA, Phipps JA, Jobling AI, Fletcher EL. Early inner retinal astrocyte dysfunction during diabetes and development of hypoxia, retinal stress, and neuronal functional loss. Invest Ophthalmol Vis Sci. 2011;52:9316-9326.

29. Hollborn M, Dukic-Stefanovic S, Pannicke T, et al. Expression of aquaporins in the retina of diabetic rats. Curr Eye Res. 2011; 36:850-856.

30. Qin Y, Fan J, Ye X, Xu G, Liu W, Da C. High salt loading alters the expression and localization of glial aquaporins in rat retina. Exp Eye Res. 2009;89:88-94.

31. Gerhardinger C, Costa MB, Coulombe MC, Toth I, Hoehn T, Grosu P. Expression of acute-phase response proteins in retinal Muller cells in diabetes. Invest Ophthalmol Vis Sci. 2005;46: 349-357.

32. Vujosevic S, Midena E. Retinal layers changes in human preclinical and early clinical diabetic retinopathy support early retinal neuronal and Müller cells alterations. J Diabetes Res. 2013;2013:905058. 